\section{Cahiers de Narratologie}

Analyse et théorie narratives

12 | 2005

Récit et éthique

\title{
Récit et éthique dans l'écriture du voyage de Camilo José Cela
}

\section{Carmen Dubois}

\section{(2) OpenEdition}

Journals

Édition électronique

URL : http://journals.openedition.org/narratologie/35

DOI : 10.4000/narratologie.35

ISSN : 1765-307X

Éditeur

LIRCES

Référence électronique

Carmen Dubois, «Récit et éthique dans l'écriture du voyage de Camilo José Cela », Cahiers de Narratologie [En ligne], 12 | 2005, mis en ligne le 20 avril 2005, consulté le 03 mai 2019. URL : http:// journals.openedition.org/narratologie/35 ; DOI : 10.4000/narratologie.35

Ce document a été généré automatiquement le 3 mai 2019.

\section{(c) $($ i) $(9)$}

Cahiers de Narratologie - Analyse et théorie narratives est mis à disposition selon les termes de la licence Creative Commons Attribution - Pas d'Utilisation Commerciale - Pas de Modification 4.0 International. 


\title{
Récit et éthique dans l'écriture du voyage de Camilo José Cela
}

\author{
Carmen Dubois
}

1 Malgré le caractère controversé, voire polémique du personnage, l'on peut toutefois avancer sans hésitation que Camilo José Cela est une des figures clés de la littérature espagnole contemporaine. Le succès de son premier roman La famille de Pascual Duarte en 1942 représente le début d'une longue et féconde carrière littéraire qui nous a laissé quelques œuvres majeures qui ont marqué l'histoire de la littérature espagnole. Parmi les plus intéressantes, citons Voyage en Alcarria -1948-, La colmena -1951-, La Cátira -1955- San Camilo 1936- 1969- Oficio de Tinieblas -1973- Mazurca para dos muertos -1983- , La cruz de San Andrés -1994-, Madera de Boj - 1999-.

2 Aussi son œuvre s'est vu récompensée par de nombreux prix littéraires nationaux (citons le Prix National de Littérature -1983, Mazurca para dos muertos-, le Prix Principe de Asturias des Lettres- 1987-, le Prix Planeta - 1994, La cruz de Santa Andrés- , le Prix Cervantes -1995- ) et elle a obtenu en 1989 la reconnaissance suprême, le Prix Nobel de Littérature.

3 Parmi sa vaste production littéraire, ses nombreux récits de voyage ont été longtemps méconnus du public et délaissés par la critique. En dehors de Voyage en Alcarria, les récits postérieurs ont connu un succès éditorial modéré et ont été l'objet de jugements plutôt sévères.

$4 \quad$ Pourtant il me semble que dans les récits de voyage de Cela l'on peut trouver une des clés de lecture de l'ensemble de son oeuvre. Tout d'abord, parce qu'il s'agit d'un véritable cycle littéraire, ce qui permet à l'auteur de créer un univers cohérent et porteur de sens. Le cycle s'ouvre par Viaje a la Alcarria en 1948 et prend fin en 1986 avec Nuevo Viaje a la Alcarria. Durant ces trente huit ans qui séparent le premier et le dernier récit, il publie Del Miño al Bidasoa, Cuaderno de Guadarrama, Avila, Judíos, moros y cristianos, Primer Viaje Andaluz, et Viaje al Pirineo de Lérida.

5 Ensuite, parce que dans son écriture du voyage, Cela paraît se dévoiler plus ouvertement que dans sa production romanesque. Il est vrai que l'allure lente du voyage à pied, qui gagne le rythme du récit lui-même, est propice à la pause, à la méditation, aux 
confidences. Ainsi, dans les récits de voyage de Cela il ne se passe presque rien. Le lecteur ne doit pas s'attendre à un récit d'aventures : les lieux parcourus n'ont rien d'exotique, le voyeur n'accomplit pas des gestes héroïques. L'important est ailleurs, dans les émotions du voyageur, dans ses réflexions, dans ses rapports avec les gens qu'il rencontre, dans son appréhension du monde qui l'entoure.

En fin de compte, le voyage - ou plutôt le vagabondage, pour utiliser un terme cher à l'auteur- s'avère être un voyage intime, un voyage à la découverte de soi. Dans ce sens, le vagabondage acquiert une dimension éthique fondamentale, car cette expérience va façonner la vision du monde et de soi-même du voyageur. Les règles de conduite qui vont gouverner les rapports du voyageur et avec le monde et avec soi-même, découlent de cette conception vitale.

7 La dimension philosophique du vagabondage n'échappe pas à Cela qui déclare dans un prologue à Viaje a la Alcarria :

Le vagabondage n'est pas un sport mais un état d'âme et même une façon d'être, une manière définitive ou irrévocable de concevoir tout ce qui survient. Lorsqu'un homme prend la route, sans but précis, il exprime, même s'il ne le veut pas et sans en être conscient, une déclaration de principe, une confession de foi, qui n'est pas moins réelle parce que fondée sur le principe d'opposition. Par son départ, l'homme qui prend la route proteste contre le fait que le monde va mal, bien que lui-même, probablement, n'aille pas beaucoup mieux. C'est un jeu compliqué dans lequel on ne comprend presque rien car presque rien n'est exprimé. ${ }^{1}$

Le vagabondage impliquerait ainsi une attitude particulière face aux événements et aux gens et disposerait d'un véritable code éthique à l'usage des voyageurs-vagabonds puisqu'il se propose comme une certaine vision du monde. Bien qu'incontestables, les principes de ce code restent implicites. C'est au fil des récits de voyage -à travers des propos tenus par le narrateur ou le voyageur, à travers des actes et des attitudes-que les lois du vagabondage peuvent être dévoilées. C'est la tâche que je me suis donnée : tenter de décrypter le code de conduite, l'éthique du voyageur Célien.

Mais revenons au point de départ, intéressons-nous à ce qui constitue le fondement même de l'expérience voyageuse. Autrement dit, demandons-nous pourquoi le voyageur, un jour, décide-t-il de prendre la route?

Dans les incipit des récits, nous découvrons un homme qui va mal, un homme qui ne semble pas trouver sa place dans le monde où il vit. Son départ s'apparente à une tentative d'échapper à un état de socialisation vécu comme une condamnation. Ce sentiment d'insatisfaction, de manque, peut prendre différentes formes: il peut se manifester tantôt physiquement -c'est le cas du récit Del Miño al Bidasoa où le voyageur part retrouver sa santé perdue-, tantôt moralement. C'est sans doute dans le dernier récit que le sentiment de mécontentement est exprimé avec plus d'intensité, voire de brutalité :

Le voyageur s'apprête à commencer ce nouveau périple complètement las de tout, bon, on ne doit jamais être excessif, disons plutôt légèrement las de tout : de la famille, du courrier, du télégraphe, du téléphone, des poètes et des romanciers, des éditeurs et des traducteurs, des peintres et des dessinateurs, des journalistes et des préfaciers, des professeurs et des académiciens, des louangeurs et des critiques, des civils, des militaires et des hommes d'église, des sociologues et des politiciens, des économistes et des retraités, des cadres dynamiques et des pensionnaires résignés, etc... ${ }^{2}$ 
11 C'est pourquoi, le simple fait de partir, de prendre la route est vécu comme une délivrance, le chemin étant un baume pour l'âme :

Le chemin est le repos du vagabond, le réconfortant viatique de celui qui est inquiet

et aussi l'eau miraculeuse qui convient au baptême de l'avanie et de la fatigue ${ }^{3}$

12 Mais le voyage n'est pas que bénédiction et bonheur, il est aussi exigence et difficulté. Avant tout, il impose au voyageur le renoncement; il doit se détacher des choses et des personnes auxquelles il tenait. Il doit ainsi rompre tout lien social : il abandonne son travail, sa famille, ses amis. C'est dans ce sens qu'il faut interpréter l'anonymat du protagoniste: désigné successivement par le syntagme " le voyageur " puis par "le vagabond", il se trouve privé de tout statut social. Ce glissement sémantique cache une amplification du procès d'isolement. En supprimant les attaches sociales, il devient un homme marginal.

13 Le voyage exige aussi le dépouillement physique, matérialisé par les bagages emportés, réduits au strict minimum: des chaussures de marche, une gibecière, une gourde et quelques victuailles.

14 Abandonnant ainsi un certain confort, sa nouvelle existence s'inscrit sous le signe de l'austérité, voire de la privation. Ces repas, très frugaux, ne varient pratiquement jamais et se limitent à un morceau de pain, quelques fruits, un peu de viande séché et une gorgée de vin.

15 Si la nuit il trouve refuge dans de modestes auberges, il lui arrive parfois de dormir à la belle étoile.

Le voyage, qui doit se faire à pied, suppose une fatigue physique considérable ; les longues heures de marche, la chaleur, le relief parfois escarpé épuisent le voyageur.

Par ailleurs, il est condamné à la solitude ; bien qu'il croise de nombreux personnages, il continue son voyage toujours seul car il ne doit pas s'attacher, créer des liens avec eux. Cela mettrait en danger son voyage.

18 Se séparer des gens pour s'enfermer dans sa solitude ne vas pas toujours sans souffrance le voyageur est un homme sensible aux gens, qui aime la compagnie; ainsi il doit se faire violence pour se désintéresser de Sindo Gallineja, enfant abandonné a son sort qu'il croise dans un village castillan :

Le vagabond, qui aime la solitude, parfois est effrayé par la solitude. Si la solitude ne se brisait pas avec le regard de Sindo, le vagabond l'aurait pris par la main. Mais la solitude est jalouse, voire tyrannique et Sindo Gallineja resta seul, pendant que le vagabond, probablement résigné, reprit son chemin sans retourner la tête afin de ne pas se transformer en statue de sel. ${ }^{4}$

19 S'il s'interdit de dormir plus de deux nuits au même endroit et de s'attacher aux gens, c'est qu'un grand danger le guette sans relâche : la sirène du confort, l'attrait de la facilité de la vie sédentaire :

Le chemin a été fait pour avancer et le fait de s'asseoir au bord du chemin, pour parler avec les gens, finit par corrompre. ${ }^{5}$

Cependant les privations, la fatigue, l'isolement inhérents au voyage, loin d'être vécus comme des contraintes, semblent se métamorphoser en principe de plaisir et de ressourcement:

21 Le voyageur étale sa couverture et se couche au bord de la route, au pied d'une aubépine. Il ne fait pas froid. La nuit est calme et étoilée. Une chouette hulule du haut d'un olivier et 
une sauterelle chante entre les chardons. Le voyageur, qui est fatigué, s'endort vite d'un sommeil calme, profond et réparateur...

Il se réveille quand il fait encore nuit, il boit une gorgée de vin, il mange deux oranges et un morceau de pain, et il commence à marcher se sentant sans doute plus fort que jamais, sans sentir le poids de la gibecière, ni de ses jambes, ni la difficulté du chemin. ${ }^{6}$

Il en retire même une satisfaction qui s'apparente au bonheur :

Mais le vagabond, qui sait être heureux à force de ne rien demander, absolument rien, a tordu le cou à l'aveugle taupe des caprices, toujours si inutiles, il y a si longtemps qu'il ne s'en souvient pas. ${ }^{7}$

24 En fin de compte, ce renoncement se présente comme une première étape, un passage obligé pour accéder à une forme de bien-être. Indifférent aux biens matériels, n'exigeant presque rien de la vie, il peut s'adonner aux petits bonheurs de l'existence, aux plaisirs élémentaires dont il sait récolter un plaisir intense et profond.

On assiste ainsi à une nouvelle forme de bonheur qui passe avant tout par la réhabilitation du corps, en tant que source de plaisir. Cette nouvelle vision du corps traduit une conception du monde positive et vitaliste fort bien résumé dans ce passage de Viaje al Pirineo de Lérida :

La vie est un cadeau que les dieux ont fait à l'homme : ils ont oublié de lui exiger de ne pas l'hypothéquer. La vie a été inventée pour vivre et pour laisser vivre, pour marcher, pour aimer les femmes que l'on croise sur la route (la tresse dénouée et la jupe soulevée), pour manger du bon pain et du jambon séché, pour boire l'eau des sources et le vin des pressoirs, pour voir le monde et parler des récoltes et des navigations, pour se baigner au bord de la rivière qui descend de la montagne et se sécher ensuite au soleil, sur l'herbe. ${ }^{8}$

Le voyageur va donc laisser ses sens explorer le monde qui l'entoure; les impressions sonores et olfactives deviennent une source intarissable de plaisirs. D'ailleurs, il proclame sans ambages son approche sensorielle de la réalité :

Comme je dispose de beaucoup de temps, j'aime m'attarder sur les choses, les regarder à l'endroit et à l'envers, les voir le matin, les sentir l'après-midi et les toucher le soir. ${ }^{9}$

Sa vie semble rythmée par l'assouvissement des besoins les plus élémentaires, tels que calmer sa faim, se désaltérer, se reposer, soulager son ventre ou sa vessie.

La nourriture et la boisson occupent une place de choix dans sa vie. Oubliant vite les maigres repas de sa première sortie, le voyageur se livre allègrement aux plaisir de la table. Une grande partie des scènes consacrées aux repas, affichent le même goût de la démesure, la même propension à la surabondance. On peut parler de caractère gargantuesque dans la description de certains repas :

Le voyageur dîne [...] un plateau de fruits de mer, varié et abondant, chose presque inconcevable dans un endroit aussi éloigné de la mer, avec des huîtres et des coques, des crevettes, des pousse-pied, des étrilles et des araignées de mer, le tout accompagné d'un vin blanc de Cambados bien froid. Oh combien de sagesse et de bonne grâce, oh combien de miséricorde et de bonnes manières! Quand les fruits de mer ne firent que simple souvenir, de la cuisine sortirent encore, en rang et sans se bousculer, un agneau au four mémorable, un ragoût de perdrix digne d'un roi et, en dessert, du riz au lait aux raisins, à la cannelle et à la vanille ; l'agneau et la perdrix sont descendus accompagnés d'un Rioja d'une bonne année et le dessert fut couronné par un porto à l'arôme ancien, au palais velouté et à la couleur brune. ${ }^{10}$ 
excès alimentaires sont impressionnants, ses débordements éthyliques ne sont pas en reste. Ainsi, son parcours castillan se termine par une " intoxication éthylique" dont il mettra quinze jours à se rétablir.

La nourriture et la boisson, omniprésents dans les récits, sont aussi matière à réflexion et sujet de commentaire. Le narrateur multiplie les références aux bienfaits du vin et aux méfaits de l'eau.

31 On apprend ainsi que les vertus du vin sont innombrables ; tantôt il est assimilé à une substance nourricière et vivifiante, tantôt à un élixir miraculeux qui réjouit les cœurs mélancoliques.

Le vin berce la tête en effaçant les mauvaises pensées, il caresse la gorge en adoucissant les voix irritées, il flatte les papilles gustatives en relevant la saveur des mets exquis et apaise le cœur en éloignant les mauvais sentiments. ${ }^{11}$

ardent plaidoyer en faveur du vin contraste avec une critique de l'eau qui s'avère, selon le voyageur, dangereuse pour la santé ; l'eau provoquerait "des rhumatismes, le typhus, les vésanies et d'autres maladies du corps et de l'âme, comme les vers et la rougeole et la paralysie" 12 savoir :

" enlever les chassies, se démêler la barbe, se coiffer les cheveux de la tête, se laver

les pieds au moins à l'occasion des équinoxes et des solstices, etc. ${ }^{13}$

fois, le fait de manger et de boire n'est pas un simple besoin physiologique, il acquiert dans les récits une portée symbolique: le partage du pain et du vin devient l'image de l'amitié et de la confraternité. C'est ainsi que, lorsque Martin Díaz -charretier de Trijueque- est enfin convaincu que le voyageur est un homme de confiance, il lui offre son pain et des oignons pour lui montrer son estime.

parcours par terres castillanes, le lecteur peut constater cet axiome :

Les jeunes hommes et les jeunes filles de Matamala, de Cañicosa, de Cillamayor y de Matamorisca, défilent par petits groupes en chantonnant le refrain 'Jalisco nunca pierde'

Ils ont bien mangé et ils ont bien bu et, contents et enjoués, ils disparaissent pour se poursuivre et s'aimer entre les chênes solennels et les hermétiques et accueillantes pierres, millénaires et fécondes..$^{15}$

érotisme occupe une place importante dans la vie du voyageur; il peu revêtir des formes différentes qui vont de l'allusion discrète à l'évocation crue des ébats amoureux, en passant par l'éloge de la beauté féminine et les rêves érotiques.

Dans les premiers récits le narrateur suggère plus qu'il ne dit; la censure y est sûrement pour quelque chose. La sensualité est donc recréée par des allusions ou par l'introduction d'éléments évocateurs.

Lors de son passage à Brihuega, le voyageur semble troublé et séduit par un groupe de jeunes filles qui lavent leur linge dans le lavoir. Le thème des jeunes filles lavant des chemises - image archétypique qui symbolise, dans la poésie traditionnelle de nombreux 
pays, l'intimité érotique- imprègne la scène de sensualité. L'érotisme y est intensifié par la présence de la fontaine - lieu propice à la rencontre des amants- ainsi que par l'attitude du bouc - qui incarne la virilité- et la description de l'accouplement de deux chiens qui semblent concrétiser le désir du protagoniste :

Deux chiens s'aiment en plein soleil, avec entêtement, avec violence, avec insolence. Une poule traverse, entourée par des poussins jaunes comme le blée. Un bouc pointe dans la rue, la tête redressée, le regard profond, les cornes fières et défiantes. Le voyageur regarde, une dernière fois, les lavandières, il se lève et il s'en va. Le voyageur est un homme qui mène une vie pleine de sacrifices. ${ }^{16}$

La beauté féminine est célébrée par le narrateur et par le héros en faisant appel à tous les registres possibles. Le lyrisme contenu des passages consacrés aux jeunes filles - lavandières, paysannes -, laisse place à un ton plus cru «si les culs de la femme andalouse ont attiré son attention ce n'est pas, spécialement, par leurs défauts et leurs imperfections, mais bien au contraire, par leurs nombreuses et manifestes qualités, à savoir : la fermeté, la pétulance, la vivacité et j'en passe. " 17

Les fréquents rêves érotiques du héros témoignent de l'importance que la sexualité a dans sa vie. Souvent ils servent d'exutoire à des impulsions réprimées dans la journée ; dans ce sens, ils libèrent les tensions du héros. La sensualité d'une situation concrète provoque la rêverie érotique qui sera interrompue brusquement, empêchant ainsi l'accomplissement de l'acte :

Après deux jours et deux nuits passées à flâner et à regarder -Oh mon Dieu, Catalina! - les jeunes filles de Candeleda, les femmes les plus belles de tout le royaume [...] il rêva de Catalina, la torera, et de son châle et de sa robe de dimanche. Quand Catalina, la torera, elle si fougueuse, si belle, si gentille et si amoureuse [...] allait dire oui, pourquoi pas, un camion inconsidéré effaça de sa tête ses intentions galantes en même temps qu'il faisait fuir, d'un grondement sourd, le bienheureux rêve du vagabond. C'est bien dommage ! 18

43 Le héros ne se contente pas toujours du rôle de simple spectateur. Lorsque l'occasion se présente, il s'adonne avec volupté aux plaisirs de la chair ; ainsi, il s'attardera trois jours et trois nuits dans la bienveillante solitude de la campagne catalane, en compagnie d'une séduisante bergère.

44 Le visage charnel de l'amour semble être la seule facette retenue dans les récits; qu'il s'agisse d'ébats animaux et humains ou encore de rêves érotiques, l'acte sexuel revêt un caractère primitif et sauvage. Intimement lié à la nourriture et à la boisson, il correspond à un besoin primaire au même titre que la nécessité de calmer la faim ou d'étancher la soif.

Ne négligeant aucun besoin élémentaire, la narration souligne à plusieurs reprises le caractère obligatoire des actes physiologiques garants d'une bonne santé. Le style peut être concis et pondéré mais, dans la plupart des cas, il utilise un ton délibérément cru et provocant:

Le vagabond, dans le profond pot de chambre émaillé caché, parmi d'autres trésors, dans la table de nuit, il pissa comme un polonais. Quelle délicieuse bénédiction! Le vagabond, qui connaît fort bien les règles pour être en bonne santé, se souvient, de temps en temps, de cette honorable pensée hippocratique : pisse simplement, pète fort et emmerde la mort ${ }^{19}$

Cet acte physiologique se trouve, en quelque sorte, ennobli par le cadre où il a lieu. L'adoption de la nature comme lieu de prédilection pour satisfaire les besoins naturels, n'est pas un hasard; l'immense solitude d'un paysage, la contemplation de minuscules 
animaux qui, indifférents à la présence humaine, poursuivent leur chemin, semblent inspirer notre voyageur :

Dans le paysage il n'y a pas la moindre trace de l'homme, pas de berger, ni de pêcheur, ni de chasseur, ni de baigneur du dimanche; sur la terre brune ou dans l'herbe parsemée de fleurs de toutes les couleurs (les jaunes dominent) on ne voit ni crotte de mouton, ni excrément de chèvre, ni déjection d'âne, ni bouse de vache, ni fèces de chrétien, et le voyageur pense, un instant, s'il ne se trouve pas au bout du monde. Le voyageur, devant une telle solitude, devant un isolement quasi métaphysique, s'assoit sur trois notions abstraites, l'infini, la nécessité et l'impunité, et il fiente avec autant de joie charnelle que de paix dans l'âme et de tranquillité de conscience parce qu'il sait bien que la crotte d'un galicien ne pollue pas car elle est biodégradable. ${ }^{20}$

Pour compléter le répertoire des besoins élémentaires du héros, nous devons nous intéresser au repos, activité à laquelle il consacre une grande partie du temps de son existence vagabonde. Si le vagabond se réserve des instants de répit pour reprendre des forces afin de poursuivre sa route, les temps d'arrêt représentent, avant tout, des instants privilégiés pour savourer la vie, prendre le temps de contempler et d'apprécier le monde qui l'entoure ainsi que pour méditer, faire le point, ou simplement rêvasser en laissant libre cours à son imagination.

La sieste est un des moments préférés du vagabond. Après avoir étanché sa soif et assouvi sa faim, il aime à s'abandonner doucement, paisiblement, tel un enfant à la conscience tranquille, jusqu'à ce qu'il tombe dans un sommeil réparateur, source d'un bien-être profond.

49 Si la nuit, la fatigue aidant, le vagabond trouve aisément un sommeil placide, profond et régénérateur, le jour, il consacre de longs moments à la contemplation de la nature ou des hommes. Il proclame haut et clair que l'oisiveté fait partie de l'art de vivre qu'est le vagabondage ; le héros, se doit d'observer ce précepte s'il veut honorer la noble famille à laquelle il dit appartenir.

Loin d'être une contrainte, l'inactivité s'avère un présent de Dieu, réservé à un petit groupe d'initiés - dont le vagabond fait partie - qui sont les seuls capables d'apprécier pleinement les plaisirs simples mais intenses de cette occupation, de «cette bénédiction que l'homme ne sait pas savourer, délicieusement, lentement, tranquillement, allongé sur le bas côté, la gourde à portée de main, la cigarette à la bouche et la tête peuplée d'imprécis, incertains et bien dessinés oiseaux volants "21

51 Il ne fait plus de doute que le voyageur fait preuve d'une approche sensorielle de la réalité et qu'il en a fait un " art de vivre" dont il retire un plaisir vif et total.

52 Cette attitude concourt à la réhabilitation du corps et, tout particulièrement, des fonctions naturelles. En même temps, elle me semble révélatrice d'une conception de l'existence qui, en exaltant les bonheurs simples et primaires, se veut proche d'une nature conçue comme source de connaissance :

La cigogne, la chouette et l'hirondelle sont les trois oiseaux qui ont appris au vagabond les choses les plus utiles. La cigogne initia le vagabond à l'art de ne se laisser voir que par périodes (...) La chouette apprit au vagabond à ne pas sourciller et il devint, à force de courage, maître dans le difficile art de la patience. L'hirondelle lui inculqua la saine prédisposition à chanter, quoiqu'il arrive, comme un fou et sans demander la permission. Le vagabond apprit aussi du moineau à faire contre mauvaise fortune bon cœur; du frisquet à dormir par terre, de la bergeronnette à vivre de l'air, entre deux gorgées d'eau..$^{22}$ 

lieu de purification et de rédemption. Le contact avec les éléments naturels devient, dès lors, primordial pour le vagabond qui s'évertue à atteindre une véritable communion avec cet objet de vénération qu'est la nature. le voyageur se sent purifié. L'eau partage avec la terre la puissance régénératrice. L'on retrouve dans les récits plusieurs motifs qui renvoient à la symbolique de la purification et du renouveau.

texte consacré intégralement au bain dans l'eau douce, l'auteur met en relief le caractère rituel de cet acte qui est, par ailleurs, réservé à un certain type d'homme :

Le bain d'eau douce, le bain d'eau paisible, d'eau nourricière, d'eau tendre et champêtre, est toujours un bain originel et baptismal, un bain liturgique et saisissant, un bain que l'on doit prendre l'âme nue comme à l'annonce du pardon. Celui qui ne se sent pas libre du péché de la civilisation, ne doit pas se dévêtir dans le vert et tendre rivage. ${ }^{23}$ lui apporte un bonheur parfait ; ainsi, sur les bords du Guadalquivir, entouré d'animaux, en contact étroit avec la terre, l'air et l'eau - matières élémentaires-, il éprouve une émotion intense qui ressemble à l'extase.

J'ai essayé de montrer que le vagabondage accorde une place prépondérante à la vie physique et aux fonctions physiologiques, même les plus primaires.

Ce mouvement vers le bas, qui est amorcé par la prépondérance du plan matériel et corporel, se poursuit par un rapprochement de la force productrice et régénératrice des éléments naturels et conduit, à terme, à une réhabilitation de la vie physique.

Le rabaissement est un fait profondément positif car il nous approche de la terre, symbole de vie, de rénovation et de fécondité. Les images scatologiques perdent ainsi toute acception grossière ou cynique ; les excréments, éléments à mi-chemin entre la terre et le corps, fécondent cette première se transformant ainsi en principe de vitalité et de renouvellement.

2 Les nombreux passages consacrés à la nourriture et à la boisson sont, de même, fortement dynamiques dans le sens où incarnent l'abondance croissante et inépuisable du principe matériel. La satisfaction des besoins naturels acquiert un sens rénovateur et réjouissant et procure au vagabond un sentiment de bonheur proche de la béatitude.

Dans ce sens, l'attitude vitale du voyageur peut être interprétée comme une conception festive et carnavalesque de l'existence, cette fête étant intimement liée à la vie du corps, de la terre, de la nature, du cosmos. La logique du carnaval étant celle du monde à l'envers, l'on peut glorifier les activités biologiques ainsi que les normes contraires aux canons officiels. La charge transgressive, inhérente au renversement de valeurs, qui soustend cet art de vivre qu'est le vagabondage est exprimé de façon très explicite : 
Prendre la route est une expression qui a deux acceptions : la première, simple et directe, qui signifie, ni plus ni moins, prendre le chemin ou le sentier, en fuyant la ville et les citoyens, pour écouter le chant harmonieux du chardonneret et voir naître et se coucher notre père le soleil, et une autre acception, plus recherchée, qui veut dire "prendre tout par-dessous la jambe" " ou "faire un bras d'honneur aux spectateurs" et comme dans la chirurgie de guerre, couper net. ${ }^{24}$

aspirations du voyageur - est fondée sur une critique préalable de la société qui, dans les récits, prend la forme d'une attitude méfiante envers la civilisation urbaine et industrielle.

71 Pour le voyageur la civilisation est fondamentalement urbaine. Dédaignant son contraire, elle s'est construite contre la nature car cette dernière s'oppose à la volonté de modifier un ordre dont les racines remontent aux temps immémoriaux. 


$$
\begin{aligned}
& \text { et plus vrai. Le voyageur reprend l'idée } \\
& \text { n'hésite pas à proclamer cette préférence. }
\end{aligned}
$$
certaine forme de bonheur.

83 En nette opposition avec la critique de la ville et du progrès, l'on note dans les récits un attachement durable au monde agricole et préindustriel, une véritable adoration de la nature dont la représentation se rapproche d'une image paradisiaque et idyllique. 

présent. Ce refus du monde actuel se manifeste, d'abord, par le rejet d'une civilisation où les machines et l'industrialisation triomphante compromettent fortement l'harmonie et l'équilibre qui régnaient autrefois. menaçante inhospitalière et angoissante au vagabond. Dès lors, il va se trouver une échappatoire dans l'affabulation d'un monde idéal, enraciné dans le passé, d'un monde instinctif à l'écart de la civilisation et de la vie urbaine, un monde où l'homme peut se sentir libre en accordant sa trajectoire avec celle que la nature lui fixe.

J'ai essayé de montrer le vagabondage comme un voyage philosophique qui se décline selon trois modalités complémentaires. L'expérience voyageuse se propose comme une philosophie du renoncement, de la vitalité et de l'utopie à la fois. Cette doctrine qu'est le vagabondage est régie par une déontologie très rigoureuse : le détachement, la solitude, l'empire de la vie matérielle et des sens, la communion avec la nature, la transgression des valeurs établies, l'éloignement de la ville, le refus du progrès, l'attachement au monde 
rural, la mythification du passé, la recherche d'un paradis perdu, étant les plus manifestes.

En dernière instance, les récits de voyage illustrent le thème du conflit entre l'individu et le monde, de la douloureuse recherche de la liberté individuelle. Le vagabondage, éthique exigeante et réservée à quelques êtres d'exception, se veut une voie qui mène à l'acquisition de la récompense suprême :

Le voyageur est complètement seul mais ce sentiment ne le dérange pas; il y a très longtemps que le voyageur sait que la solitude est le prix à payer pour avoir d'autres choses: l'indépendance, la paix avec soi-même, pouvoir faire un bras d'honneur au purgatoire, la liberté de traverser cette vallée de larmes sans trop de brides dans la conscience et la pensée et ainsi de suite. ${ }^{32}$

La portée de l'expérience voyageuse ne se limite pas au destin individuel du vagabond. Cet homme en perpétuelle errance qu'est le voyageur célien peut être considéré comme une allégorie du fatum de l'humanité entière, condamnée à errer sur le chemin de la vie dont l'aboutissement est immuable et sans appel. La vie est une route et l'homme un voyageur; la seule liberté que nous ayons c'est de trouver et de suivre notre propre chemin - un chemin vrai et non-conformiste-, c'est aussi notre seule gloire.

\section{NOTES}

1. Camilo José CELA. Viajes por España . Volumen I.. Barcelona : Destino, 1965, p. 519.

2. Camilo José CELA. Nuevo Viaje a la Alcarria. Barcelona : Plaza \& Janés, 1994, p. 15.

3. Camilo José Cela. op. cit., p. 228.

4. Camilo José CELA. Judíos, moros y cristianos. Barcelona : Destino, 1965, p. 148.

5. Camilo José CELA. Viaje a la Alcarria. Barcelona : Destino, 1965, p. 172.

6. Camilo José CELA. Viaje a la Alcarria. Barcelona : Destino, 1965, p. 174.

7. Camilo José CELA. Cuaderno del Guadarrama. Barcelona : Destino, 1966, p. 53.

8. Camilo José CELA. Viaje al Pirineo de Lérida. . Barcelona : Destino, 1968, p. 379.

9. Camilo José CELA. Judíos, moros y cristianos. Barcelona : Destino, 1965, p. 152.

10. Camilo José CELA. Nuevo Viaje a la Alcarria. Barcelona : Plaza \& Janés, 1994, p. 135-136.

11. Camilo José CELA. Nuevo Viaje a la Alcarria. Barcelona : Plaza \& Janés, 1994, p. 133.

12. Camilo José CELA. Del Miño al Bidasoa: Notas de un vagabundaje. Barcelona : Destino, 1965, p. 347.

13. Camilo José CELA. Viaje al Pirineo de Lérida. . Barcelona : Destino, 1968, p. 573.

14. Camilo José CELA. Primer viaje andaluz: Notas de un vagabundaje por Jaén, Córdoba, Sevilla, Huelva y sus tierras. Barcelona : Destino, 1968, p. 112.

15. Camilo José CELA. Judíos, moros y cristianos. Barcelona : Destino, 1965, p. 145.

16. Camilo José CELA. Viaje a la Alcarrria. Barcelona : Destino, 1965, p. 81.

17. Camilo José CELA. Primer viaje andaluz: Notas de un vagabundaje por Jaén, Córdoba, Sevilla, Huelva y sus tierras. Barcelona : Destino, 1968, p. 308-309.

18. Camilo José CELA. Judíos, moros y cristianos. Barcelona : Destino, 1965, p. 435.

19. Camilo José CELA. Primer viaje andaluz: Notas de un vagabundaje por Jaén, Córdoba, Sevilla, Huelva y sus tierras. Barcelona : Destino, 1968, p. 290. 
20. Camilo José CELA. Nuevo Viaje a la Alcarria. Barcelona : Plaza \& Janés, 1994, p.143.

21. Camilo José CELA. Judíos, moros y cristianos. Barcelona : Destino, 1965, p. 128.

22. Camilo José CELA. Judíos, moros y cristianos. Barcelona : Destino, 1965, p. 369-370.

23. Camilo José CELA. Cajón de sastre. Madrid : Ediciones Cid, 1957, p. 272-273.

24. Camilo José CELA. Viajes por España . Volumen I.. Barcelona : Destino, 1965, p. 514.

25. Camilo José CELA. Viajes por España . Volumen I.. Barcelona : Destino, 1965, p. 518.

26. Camilo José CELA. Viaje a l Pirineo de Léridq.. Barcelona : Destino, 1968, p. 381.

27. Camilo José CELA. Cuaderno del Guadarrama.. Barcelona : Deestino, 1966, p. 41.

28. Camilo José CELA. Cuaderno del Guadarrama.. Barcelona : Deestino, 1966, p. 40.

29. Camilo José CELA. Nuevo Viaje a la Alcarria. Barcelona : Plaza \& Janés, 1994, p.171.

30. Camilo José CELA. Viaje al Pirineo de Lérida. Barcelona : Destino, 1968, p. 456.

31. Camilo José CELA. Primer viaje andaluz. Barcelona : Destino, 1968, p. 356.

32. Camilo José CELA. Nuevo viaje a la Alcarria. Barcelona: Plaza \& Janés, p. 16.

\section{RÉSUMÉS}

Au delà de la simple narration d'un parcours spatial, les récits de voyage de Camilo José Cela ont une forte dimension éthique. Le vagabondage célien est la manifestation d'une certaine vision du monde et de l'homme construite autour de trois notions clés: renoncement, vitalité et utopie. Le voyageur se veut ainsi à l'image de cet idéal célien; sa vie est réglée par une déontologie précise : le détachement, la solitude, la communion avec la nature, l'éloignement de la ville et l'attachement au monde rural sont les plus manifestes. En dernière instance, le vagabondage, éthique exigeante et réservée à quelque êtres d'exception, est la voie qui mène à la liberté et au bonheur.

INDEX

Palabras claves : Camilo José Cela

Mots-clés : voyage, renoncement, utopie, vitalisme 